\title{
A Cost Sensitive Decision Tree Algorithm Based on Weighted Class Distribution with Batch Deleting Attribute Mechanism
}

\author{
Hong Zhao*, Xiangju Li \\ Lab of Granular Computing, Minnan Normal University, Zhangzhou 363000, China
}

\begin{abstract}
Minimal cost classification is an important issue in data mining and machine learning. Recently, many enhanced algorithms based on the C4.5 algorithm have been proposed to tackle this issue. One disadvantage in these methods is that they are inefficient for medium or large data sets. To overcome this problem, we present a cost-sensitive decision tree algorithm based on weighted class distribution with a batch deleting attribute mechanism (BDADT). In the BDADT algorithm, a heuristic function is designed for evaluating attributes in node selection. This contains a weighted information gain ratio, a test cost, and a user-specified non-positive parameter for adjusting the effect of the test cost. Meanwhile, a batch deleting attribute mechanism is incorporated into our algorithm. This mechanism deletes redundant attributes according to the values of the heuristic function in the process of assigning nodes to improve the efficiency of decision tree construction. Experiments are conducted on 20 UCI data sets with representative test cost normal distribution to evaluate the proposed BDADT algorithm. The experimental results show that the average total costs obtained by the proposed algorithm are smaller than the existing CS-C4.5 and CS-GainRatio algorithms. Furthermore, the proposed algorithm significantly increases the efficiency of cost-sensitive decision tree construction.

Keywords. cost sensitive; decision tree; deleting attribute mechanism; granular computing.
\end{abstract}

\section{Introduction}

Classification is a data mining task that necessitates the building of a model or a classifier for a given set of pre-classified examples to classify categories of new events $[1,5,6,24]$. Many classification methods have been designed in machine learning and pattern recognition, such as decision trees $[20,23,36]$,

\footnotetext{
${ }^{*}$ Corresponding author. Tel.: +86 13656045192

Email address: hongzhaocn@163.com (Hong Zhao)
}

Preprint submitted to Elsevier

September 1, 2016

(C) 2016. This manuscript version is made available under the Elsevier user license http://www.elsevier.com/open-access/userlicense/1.0/ 
rough set theory $[15,16,32,43,49]$, neighborhood rough set theory [14], artificial neural networks $[8,13,45], k$-nearest neighbor $[4,18,44]$, and other granular computing methods $[12,22,33,34]$.

Decision trees are a natural way of presenting a decision-making process because they are simple and easy for anyone to understand [35]. Hence, decision trees have a wide range of applications in business [9], manufacturing [31], computational biology [19], and so on. These methods aim at training classifiers to maximize accuracy, with all classification errors implicitly having the same cost $[7,21,41]$. In many important applications, different misclassification errors often lead to different costs $[17,40,47,48]$. Many researchers have taken cost factors into consideration in classification studies regarding decision tree models $[23,28,10]$.

Minimal cost decision tree construction is a hot topic in data mining and machine learning. Many methods are available for cost-sensitive decision tree construction, such as EG2 [26], IDX [30], CS-ID3 [38], $\lambda$-ID3 [28], CS-GainRatio [10], and ICET[39]. These methods can construct an effective cost-sensitive decision tree to minimize the misclassification cost and the test cost. However, there are still two techniques that can be improved in cost-sensitive decision tree construction. (1) The imbalance class distribution is not taken into account when constructing the cost-sensitive decision tree. Some studies consider the imbalance class distribution only when the cost-sensitive decision tree construction is finished. (2) These methods assign nodes by evaluating all attributes. However, it is not necessary to consider every attribute for node selection. This has low efficiency for medium or large data sets. For example, we need to test 20000 attributes when assigning each node in the SMK-CAN-187 data set, which contains 19993 condition attributes.

In this paper, an algorithm based on weighted class distribution with a batch deleting attribute mechanism (BDADT) is introduced for cost-sensitive decision tree construction. The main contributions of the method are twofold. (1) To ensure the effectiveness of BDADT, we take the imbalance class distribution into account when we define the new heuristic function for selecting attributes as nodes in the tree. Specifically, we balance the weighted class distribution and the test cost of attributes as the information gain ratio. This is more realistic than the existing tree construction. (2) To ensure the efficiency of BDADT, we incorporate a batch deleting attribute (BDA) mechanism to delete redundant attributes in the node selection procedure. This can reduce calculation in decision tree construction. For instance, $80 \%$ of the condition attributes are deleted in selecting the root node for the Prostate-GE data set. As another example, 13996 attributes are deleted in selecting the root nodes for the SMK-CAN-187 data set, and the decision tree contains 36 non-leaf nodes. That is, it reduces attribute evaluation by a factor of $13996 \times 35=489860$.

In addition, test cost and misclassification cost are both considered in this paper, which are two important costs in real applications. The test cost is increased and the misclassification cost is reduced when more attributes are selected. Therefore, we should select attribute subsets by considering the tradeoff between the test cost and the misclassification cost. This is more reasonable in 
several real applications such as cancer recognition and tumor classification.

Twenty data sets from the University of California-Irvine (UCI) [3] library are used to study the efficiency and effectiveness of the proposed algorithm. We adopt a representative normal distribution to generate test costs from a statistical viewpoint. Experiments are undertaken with the open source software COSER (cost-sensitive rough sets) [29]. We run two groups of experiments: 1) we test the BDA mechanism using different values of the parameter $\delta ; 2$ ) we compare our proposed algorithm with previous approaches, namely the CS-C4.5 algorithm [11] and the CS-GainRatio algorithm [10]. The experimental results indicate the following. On the one hand, the BDADT algorithm with the BDA mechanism has lower cost and a shorter running time than the method without the BDA mechanism. Therefore, the BDA mechanism is effective. On the other hand, the BDADT algorithm performs better than the CS-C4.5 algorithm and the CS-GainRatio algorithm on all selected data sets.

The rest of the paper is organized as follows. Section 2 reviews the background for this article, including decision systems with test costs and misclassification costs. In Section 3, we propose the heuristic function which serves for selecting attributes and introduce the definition of minimum cost of cost sensitive decision tree problem. Section 4 introduces the batch deleting attribute scheme and the proposed algorithm. Section 5 presents experiment schemes and provides a simple analysis of the results. Finally, Section 6 concludes this paper.

\section{Decision system with test costs and misclassification costs}

This section presents some preliminary knowledge of the paper. We first introduce the concept of a decision system and a test-cost-independent decision system. A decision system with test costs and misclassification costs is then introduced.

In data mining and machine learning, decision systems with test costs and misclassification costs are an important concept and are defined as follows.

Definition 1. [42] A decision system (DS) is the 5-tuple:

$$
D S=\langle U, C, D, V, I\rangle
$$

where

1. U: a non-empty finite set of objects called the universe,

2. C: a non-empty finite set of condition attributes,

3. $D: D=\{d\}$ is a non-empty finite set of decision attributes,

4. V: $V=\left\{V_{a}\right\}$ is a set of values for each attribute $a \in C \cup D$,

5. $I: I=\left\{I_{a}\right\}$ is an information function for each attribute $a \in C \cup D$ $\left(I_{a}: U \rightarrow V_{a}\right)$.

Definition 2. [27] A test-cost-independent decision system (TCI-DS) is the 6tuple:

$$
D S=\langle U, C, D, V, I, t c\rangle,
$$


where $U, C, D, V$ and $I$ have the same meanings as in Definition 1. tc: a test cost function (i.e. $C \rightarrow R^{+} \cup\{0\}$ ).

We adopt the test cost independent model [27] to define the test cost. That is, cost function can be represented by a vector $t c=\left[t c\left(a_{1}\right), t c\left(a_{2}\right), \cdots, t c\left(a_{|C|}\right)\right]$ and $t c(B)=\sum_{a \in B} t c(a)$ for any $B \subseteq C$.

Definition 3. [46] A decision system with test costs and misclassification costs (TM-DS) is the 7-tuple:

$$
D S=\langle U, C, D, V, I, t c, m c\rangle,
$$

where $U, C, D, V, I$ and tc have the same meanings as in Definition 2 and $m c$ : a misclassification cost matrix (i.e. $m c: k \times k \rightarrow R^{+} \cup\{0\}$ ).

A TM-DS is represented by a decision table, a test cost vector and a misclassification cost matrix, as shown in Tables 1, 2 and 3 respectively. The following example is described in detail.

Table 1: An example of decision system.

\begin{tabular}{cccccccccc}
\hline Patient & $a_{1}$ & $a_{2}$ & $a_{3}$ & $a_{4}$ & $a_{5}$ & $a_{6}$ & $a_{7}$ & $a_{8}$ & $D$ \\
\hline$x_{1}$ & 8 & 155 & 62 & 26 & 495 & 34 & 0.543 & 46 & 1 \\
$x_{2}$ & 1 & 153 & 82 & 42 & 485 & 40.6 & 0.687 & 23 & 0 \\
$x_{3}$ & 1 & 149 & 68 & 29 & 127 & 29.3 & 0.349 & 42 & 1 \\
$x_{4}$ & 6 & 183 & 94 & 0 & 0 & 40.8 & 1.461 & 45 & 0 \\
$x_{5}$ & 1 & 189 & 60 & 23 & 846 & 30.1 & 0.398 & 59 & 1 \\
$x_{6}$ & 1 & 193 & 50 & 16 & 375 & 25.9 & 0.655 & 24 & 0 \\
$x_{7}$ & 1 & 144 & 82 & 40 & 0 & 41.3 & 0.607 & 28 & 0 \\
$x_{8}$ & 1 & 151 & 60 & 0 & 0 & 26.1 & 0.179 & 22 & 0 \\
$x_{9}$ & 1 & 164 & 82 & 43 & 67 & 32.8 & 0.341 & 50 & 0 \\
$x_{10}$ & 9 & 154 & 78 & 30 & 100 & 30.9 & 0.164 & 45 & 0 \\
\hline
\end{tabular}

Table 2: An example of test cost vector.

\begin{tabular}{ccccccccc}
\hline$a$ & $a_{1}$ & $a_{2}$ & $a_{3}$ & $a_{4}$ & $a_{5}$ & $a_{6}$ & $a_{7}$ & $a_{8}$ \\
\hline$t c(a)$ & $\$ 8$ & $\$ 6$ & $\$ 3$ & $\$ 4$ & $\$ 2$ & $\$ 4$ & $\$ 5$ & $\$ 5$ \\
\hline
\end{tabular}

Table 3: An example of misclassification cost matrix.

\begin{tabular}{ccc}
\hline \multirow{2}{*}{ Actual class } & \multicolumn{3}{c}{ Predicted class } \\
\cline { 2 - 4 } & 0 & 1 \\
\hline 0 & $\$ 0$ & $\$ 50$ \\
1 & $\$ 200$ & $\$ 0$ \\
\hline
\end{tabular}


Example 1. To illustrate TM-DS clearly, we randomly select ten objects from Diabetes data set which are listed in Table 1. In this table, $U=\left\{x_{1}, x_{2}, \cdots, x_{10}\right\}$, $C=\left\{a_{1}, a_{2}, a_{3}, a_{4}, a_{5}, a_{6}, a_{7}, a_{8}\right\}$ and $D=\{0,1\}$. The test cost vector is presented in Table 2. That is, the test costs of $a_{1}, a_{2}, a_{3}, a_{4}, a_{5}, a_{6}, a_{7}$ and $a_{8}$ are $\$ 8, \$ 6, \$ 3, \$ 4, \$ 2, \$ 4, \$ 5$ and $\$ 5$ respectively. We represent the misclassification cost function by Table 3, where $\$ 50$ stands for the cost associated with an "0" class object assigned to the "1" and \$200 represents the opposite misclassification scenario. Usually, $m c_{(i, i)}=0$.

\section{Heuristic function for attribute selection}

In this section, we propose weighted information entropy, the weighted information ratio and the heuristic function for attribute selection. We then introduce the calculation method for the cost-sensitive decision tree and the definition of the minimum cost decision tree problem.

\subsection{Heuristic function for attribute selection}

$S=\{U, C, D, V, I, t c, m c\}$ is a cost sensitive decision system and $d_{i} \in D$. The weighted coefficient of $i$-class is defined as:

$$
w_{i}=\frac{|U|}{\left|U_{d_{i}}\right|},
$$

where $|*|$ represents the number of elements in $*$, and $U_{d_{i}}$ is the elements with $d_{i}$-class.

Let $\operatorname{val}(a)$ be the set of the values of attribute $a \in C$. Let $S_{v}$ be the set of the value of attribute $a$, where $v \in \operatorname{val}(a)$. Let $S_{v}\left(d_{i}\right)$ be the set in which the objects belong to $i$-class, where $d_{i} \in D$.

Let partitions of the universe $U$ introduced by $a$ and $D$ be $S=\left\{S_{1}, S_{2}, \cdots, S_{|v a l(a)|}\right\}$ and $D=\left\{D_{1}, D_{2}, \cdots, D_{|\operatorname{val}(a)|}\right\}$, respectively. The weighted probability of $S_{v}$ is defined as follows

$$
p_{w}\left(S_{v}\right)=\frac{\sum_{i=1}^{|D|} w_{i} *\left|S_{v}\left(d_{i}\right)\right|}{\left|\sum_{v=1}^{|v a l(a)||D|} \sum_{i=1}^{\mid} w_{i} *\right| S_{v}\left(d_{i}\right) \mid} .
$$

The weighted probability distribution about $S$ and $D$ can be determined as follows:

$$
\left[S, p_{w}\right]=\left[\begin{array}{rrrr}
S_{1} & S_{2} & \cdots & S_{|\operatorname{val}(a)|} \\
p_{w}\left(S_{1}\right) & p_{w}\left(S_{2}\right) & \cdots & p_{w}\left(S_{|\operatorname{val}(a)|}\right)
\end{array}\right]
$$

and

$$
\left[D, p_{w}\right]=\left[\begin{array}{rrrr}
D_{1} & D_{2} & \cdots & D_{|\operatorname{val}(a)|} \\
p_{w}\left(D_{1}\right) & p_{w}\left(D_{2}\right) & \cdots & p_{w}\left(D_{|\operatorname{val}(a)|}\right)
\end{array}\right],
$$


where $P_{w}\left(D_{i}\right)=\frac{w_{i} *\left|D_{i}\right|}{\sum_{i=1}^{|D|} w_{i} *\left|D_{i}\right|}$

The concept of information entropy is important in machine learning. We define weighted information entropy as follows.

Let $a \in C, d_{i} \in D$, partitions of the universe $U$ introduced by $a$ and $D$ be $S=\left\{S_{1}, S_{2}, \cdots, S_{n}\right\}$ and $D=\left\{D_{1}, D_{2}, \cdots, D_{m}\right\}$, respectively. The weighted information entropy of the decision system $S$ is

$$
I E W(S)=-\sum_{i=1}^{m} p_{w}\left(D_{i}\right) * \log _{2}^{p_{w}\left(D_{i}\right)}
$$

The weighted information gain of attribute $a$ is

$$
\operatorname{Gain} W(a)=I E W(S)-\sum_{v \in \operatorname{val}(a)} p_{w}\left(S_{v}\right) * \operatorname{Entropy} W\left(S_{v}\right) .
$$

The weighted information gain ratio of attribute $a$ is

$$
\text { GainRatioW }(a)=\frac{\operatorname{Gain} W(a)}{\text { SplitInformation } W(a)} .
$$

The SplitInformation $W(a)=-\psi_{v} * \log _{2}^{\psi_{v}}$ is the split information entropy of the attribute $a$, which has the same meanings as in CS-C4.5 algorithm [36].

Attribute selection is a fundamental process in decision tree induction. Our proposed algorithm employs the heuristic function based on $\mathrm{C} 4.5$ as below:

$$
f(a, t c(a))=\text { GainRatioW }(a) *(1+t c(a))^{\theta},
$$

where $t c(a)$ is the test cost of attribute $a . \theta$ is a non-positive number and it is introduced to adjust the influence of the test cost. That is, an attribute with lower test and more information takes more advantage in the choice.

\subsection{Minimum cost decision tree problem}

Cost-sensitive decision tree construction is an important problem in machine learning. Let $U$ be the test data set with $x \in U$, and let $T$ be a decision tree trained from the training data set. According to the attribute value of $x$, we can follow the path $m_{x}$ from the root node to a leaf node. Let $B_{x}$ be the attributes set of path $m_{x}$. The test cost of $x$ is $t c(x)=t c\left(S_{x}\right)=\sum_{a \in S_{x}} t c(a)$.

Let $R_{x}$ be the real class of object $x, P_{x}$ be the prediction class of $x$ by $T$. The misclassification cost of $x$ is $m c(x)=m c\left(R_{x}, P_{x}\right)$. We can obtain the total cost of $x$ is $t c(x)+m c(x)$.

Definition 4. The average total cost (ATC) of decision tree $T$ on $U$ is:

$$
A T C_{T}(U)=\frac{1}{|U|} \sum_{x \in U}(t c(x)+m c(x)) .
$$




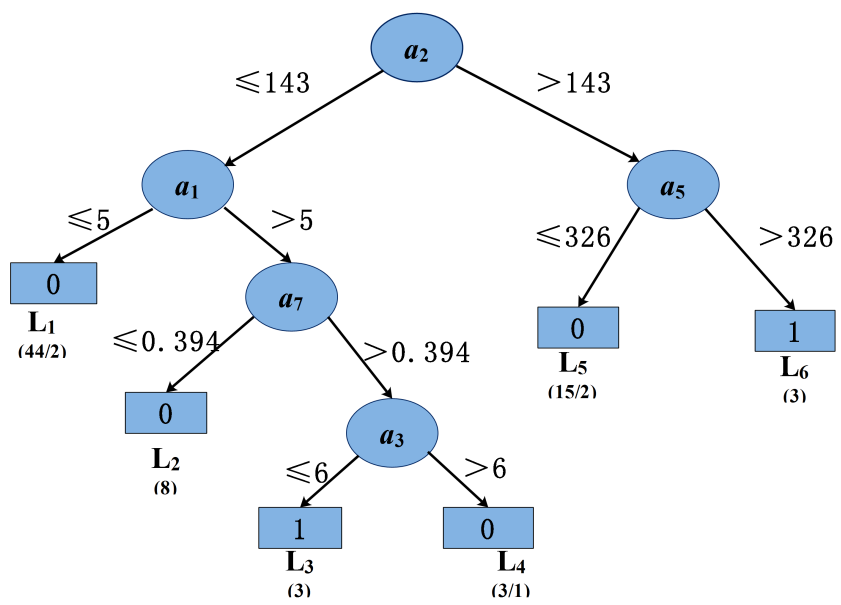

Figure 1: A cost sensitive decision tree on training data set.

The cost sensitive decision tree construction (CSDTC) problem is defined as follows.

Problem: The CSDTC problem.

Input: $S=(U, C, D, V, I, t c, m c)$.

Output: $T$, the cost sensitive decision tree.

Optimization objective: minimize $A T C_{T}(U)$.

To explain Definition 4 clearly, an example is given in Example 2.

Example 2. We randomly select a subset (10\%) for decision tree training from Diabetes. A cost-sensitive decision tree (T) obtained by the CS-C4.5 algorithm on the training data set is shown in Figure 1. Let $L$ be the set of leaf nodes: $L=$ $\left\{L_{1}, L_{2}, L_{3}, L_{4}, L_{5}, L_{6}\right\}$. The information in the rectangles represent prediction classes. The figures $(a / b)$ behind the rectangles represent two kinds information, where "a" is the number of objects in this leaf node and " $b$ " is the number of objects misclassified. For example, the information behind $L_{1}$ is "(44/2)", where "44" is the number of objects in $L_{1}$, and "2" is the number of objects whose real class is "1" but which have been misclassified as class " 0 ".

For simply, we let the objects listed in Table 1 as the testing data set $\left(U^{\prime}\right)$. Then the test cost of $x_{1}$ is: $t c\left(x_{1}\right)=t c\left(a_{2}\right)+t c\left(a_{5}\right)=\$ 6+\$ 2=\$ 8 . \quad x_{1}$ is classified as " 1 " class by $T$ and the real class of $x_{1}$ is "1". Therefore, the misclassified cost of $x_{1}$ is: $m c\left(x_{1}\right)=\$ 0$. We can get the total cost of $x_{1}$ is: $t c\left(x_{1}\right)+m c\left(x_{1}\right)=\$ 8$. Similarly, $t c\left(x_{2}\right)+m c\left(x_{2}\right)=\$ 8+\$ 50=\$ 58 ; t c\left(x_{3}\right)+$ $m c\left(x_{3}\right)=\$ 8+\$ 200=\$ 208 ; t c\left(x_{4}\right)+m c\left(x_{4}\right)=\$ 8+\$ 0=\$ 8 ; t c\left(x_{5}\right)+m c\left(x_{5}\right)=$ $\$ 8+\$ 0=\$ 8 ; t c\left(x_{6}\right)+m c\left(x_{6}\right)=\$ 8+\$ 50=\$ 58 ; t c\left(x_{7}\right)+m c\left(x_{7}\right)=\$ 8+$ $\$ 0=\$ 8 ; t c\left(x_{8}\right)+m c\left(x_{8}\right)=\$ 8+\$ 0=\$ 8 ; t c\left(x_{9}\right)+m c\left(x_{9}\right)=\$ 8+\$ 0=\$ 8 ;$ 
$t c\left(x_{10}\right)+m c\left(x_{10}\right)=\$ 8+\$ 0=\$ 8$. So, $A T C_{T}\left(U^{\prime}\right)=\frac{1}{\left|U^{\prime}\right|} \sum_{x \in U^{\prime}}(t c(x)+m c(x))=$ $\frac{1}{10} *(\$ 8+\$ 58+\cdots+\$ 8)=\$ 20$.

\section{A cost sensitive decision tree algorithm based on weighted class distribution with batch deleting attribute scheme}

In this section, a BDA mechanism is designed for node selection. A costsensitive decision tree algorithm based on weighted class distribution with BDA (BDADT) is then proposed.

\subsection{Batch deleting attribute scheme}

An outline of the BDA mechanism in our proposed algorithm is shown in Algorithm 1. This framework gives us a detailed description of the BDA mechanism for node selection, and contains four main steps.

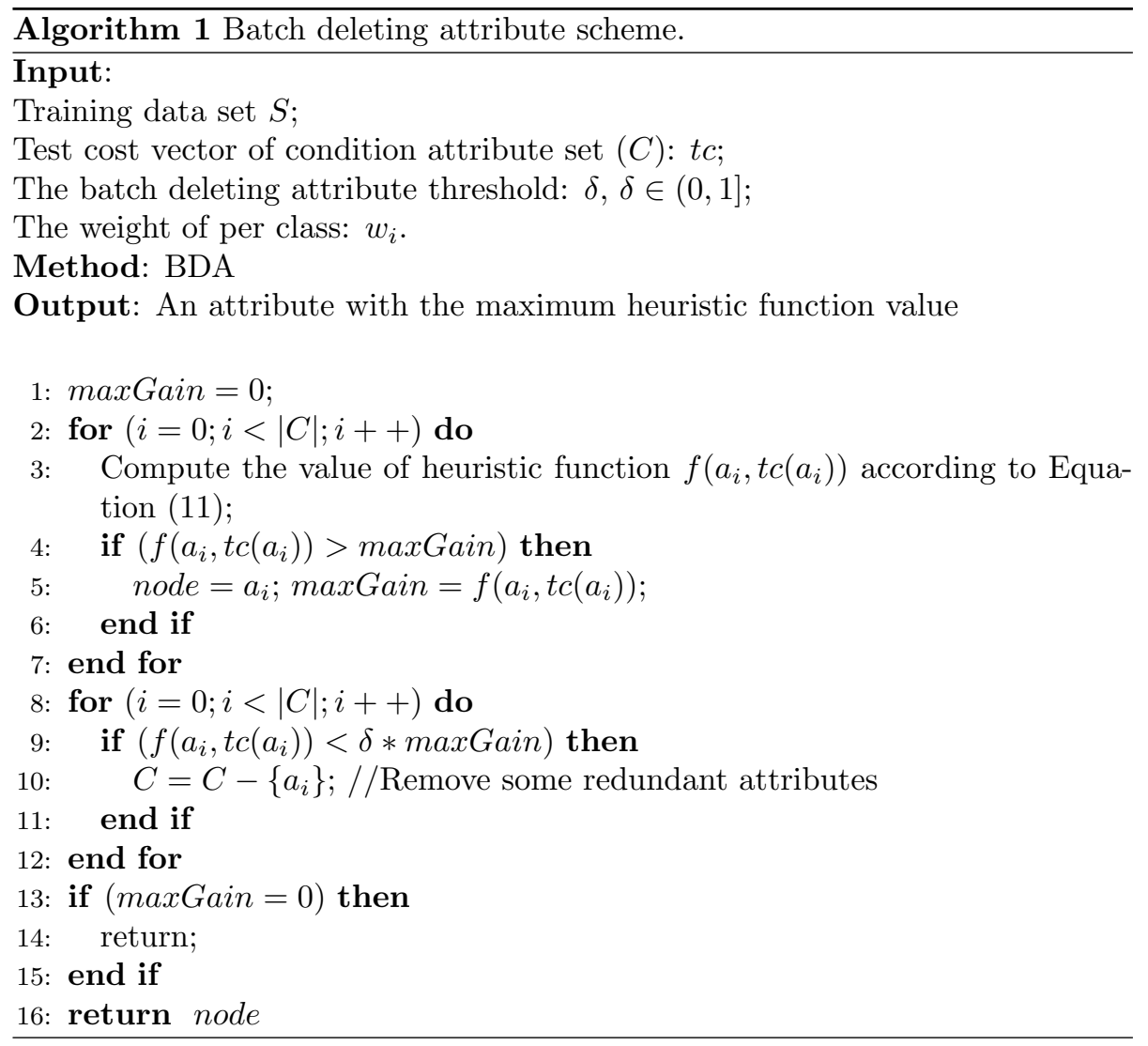

Step 1 corresponds to line 3. We compute the heuristic function value of attribute $a$ according to Equation (11). 
Step 2 corresponds lines 4 to 6 . In this step, we compare the heuristic function value of attribute $a_{i}$ with the current maximum heuristic value maxGain. If $\max G$ ain $<f\left(a_{i}, t c\left(a_{i}\right)\right)$, then we replace maxGain by $f\left(a_{i}, t c\left(a_{i}\right)\right)$.

Step 3 corresponds to lines 8 to 12 , which contain the key code of Algorithm 1. In this step, some redundant attributes are deleted and are not considered in the next node selection.

Step 4 corresponds to lines 12 to 15 . Algorithm 1 returns null if $f(a, t c(a))=$ 0 for each attribute in $C$.

An example is presented in Example 3 to explain the proposed deletion of redundant attribute mechanisms.

Table 4: Heuristic function values of attributes on Diabetes data set.

\begin{tabular}{|c|c|c|c|c|c|c|c|c|}
\hline$a$ & $a_{1}$ & $a_{2}$ & $a_{3}$ & $a_{4}$ & $a_{5}$ & $a_{6}$ & $a_{7}$ & $a_{8}$ \\
\hline $\bar{f}$ & 0.0289 & 0.0350 & 0.0071 & 0.0076 & 0.0268 & 0.0822 & 0.0045 & 0.0357 \\
\hline
\end{tabular}

Example 3. Consider the process of selecting the root node on Diabetes data set which contains 8 condition attributes and 768 objects. Table 4 lists the heuristic function values for every attribute on Diabetes data set. In this example, we let $\delta=0.5$. Firstly, we initialize the max value of heuristic function as maxGain $=0$. Then, we compute $f\left(a_{1}, t c\left(a_{1}\right)\right)$ according to Equation (11) and the heuristic function value of attribute $a_{1}$ is listed in the second column. Now, let maxGain $=f\left(a_{1}, t c\left(a_{1}\right)\right)$ since $f\left(a_{1}, t c\left(a_{1}\right)\right)=0.0289$ which is bigger than maxGain. Thirdly, compute the heuristic function value of $a_{2}$ and compare $f\left(a_{2}, t c\left(a_{2}\right)\right)$ with maxGain. As can be seen from Table 4, $f\left(a_{2}, t c\left(a_{2}\right)\right)=0.0350>\operatorname{maxGain}$. Now maxGain is refreshed, that is maxGain $=f\left(a_{2}, t c\left(a_{2}\right)\right)=0.0350$. Fourthly, we compare $f\left(a_{3}, t c\left(a_{3}\right)\right)$ with maxGain. According to BDA mechanism, attribute $a_{3}$ is deleted since $f\left(a_{3}, t c\left(a_{3}\right)\right)<\max G a i n * \delta$. Continue this process until the last attribute.

4.2. A cost sensitive decision tree algorithm based on weighted class distribution with $B D A$

In this section, we provide a detailed description of the BDADT algorithm. Algorithm 2 lists the pseudo code for the process of decision tree construction in the BDADT algorithm. It contains four main steps.

Step 1 is contained in lines 1 and 2 . We compute the size of each class and obtain the weight of each class.

Step 2 corresponds to lines 3 to 6 . An initial tree is constructed in this step. If the objects are all of the same class or there no remaining attributes for the objects to be partitioned further, then we let the current node be a leaf.

Step 3 corresponds to lines 7 and 8 . This step contains the key code for Algorithm 2. We select the best attribute as a node and delete some attributes with lower information using the BDA mechanism outlined in Algorithm 1.

Step 4 is contained in lines 9 to 12 . The branches are created according the values for the selected node. The data set is then divided into sub-data sets $S_{i}$. 


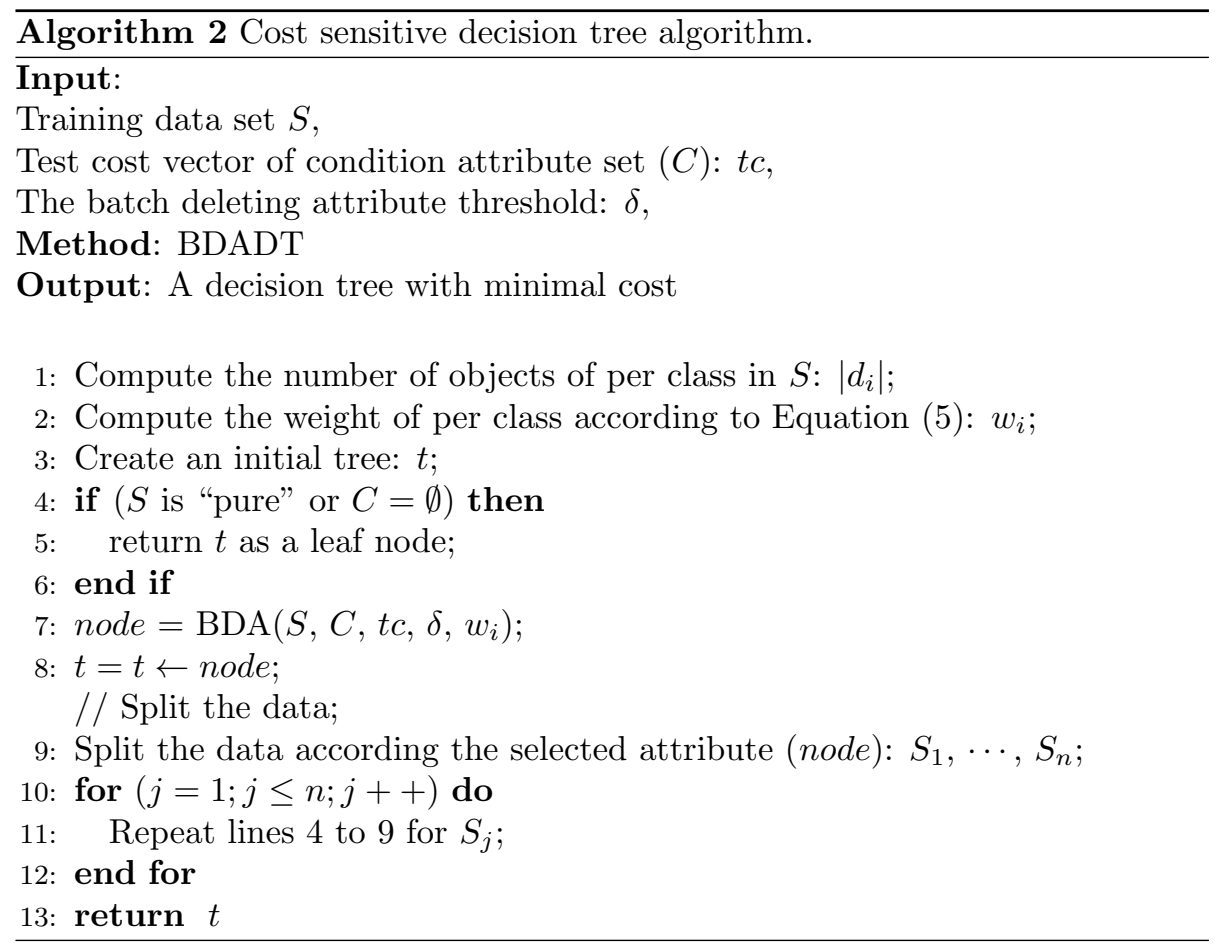

The algorithm recursively applies lines 3 to 12 to generate a decision tree for these sub-data sets.

The next problem is how to label a leaf node that has both positive and negative examples. In our method, let $L$ be a leaf node, and let $P(N)$ be the set of positive (negative) objects. The criterion is presented as:

$$
\left\{\begin{array}{lll}
P, & \text { if } & |P| * m c(P, N)>|N| * m c(N, P) \\
N, & \text { if } \quad|P| * m c(P, N)<|N| * m c(N, P)
\end{array}\right.
$$

where $|P|(|N|)$ is the number of objects in $P($ or $N)$. And $m c(P, N)(m c(N, P))$ is the cost of misclassified $P$ (or $N$ ) as $N$ (or $P$ ).

The following example introduces the implementation process of Algorithms 1 and 2 .

Example 4. Consider the Sonar data set, which contains 60 condition attributes and 208 objects. There are two classes named Mine and Rock in this data set. We select 60\% (125 samples) as the training data set, where the numbers in the Mine and Rock classes are 15 and 110, respectively.

(1) We initialize for Algorithm 2 as follows. The misclassification costs are 50 and 500, and $\lambda=-1$ and $\sigma=0.25$.

(2) Compute the weighted coefficients for Algorithm 2. The weighted coeffcient of the Mine class is $w_{1}=\frac{125}{15}$. The weighted coefficient of the Rock class is $w_{1}=\frac{125}{110}$. 
(3) Execute Algorithm 1 to compute the heuristic function value of attribute a according to Equation (11). Then select the node $a_{\max }$ with the maximum heuristic function value and delete attributes smaller than the threshold. Partial results are listed in Table 5. In this table, $a_{6}, a_{7}$, and $a_{60}$ are deleted. Return $a_{\max }$ to Algorithm 2.

(4) In Algorithm 2, split the data according the selected attribute ( $\left.a_{\max }\right)$. Then repeat the above three steps until $\operatorname{maxGain}=0$.

Table 5: The value of Quality of attribute on Sonar data set.

\begin{tabular}{cccccccccc}
\hline$a$ & $a_{1}$ & $a_{2}$ & $a_{3}$ & $a_{4}$ & $a_{5}$ & $a_{6}$ & $a_{7}$ & $\ldots$ & $a_{60}$ \\
\hline$f(a, t c(a))$ & 0.017 & 0.022 & 0.011 & 0.038 & 0.019 & $\mathbf{0 . 0 0 7}$ & $\mathbf{0 . 0 1 0}$ & $\ldots$ & $\mathbf{0}$ \\
\hline
\end{tabular}

\section{Experiments}

Twenty standard data sets with two categories are used to study the efficiency and effectiveness of the proposed BDA mechanism and BDADT algorithm. ColonTumor [2] has 62 samples collected from colon-cancer patients and 2000 attributes. SMK-CAN-187 [37] is a benchmark microarray based gene expression database. The other 18 data sets are from the UCI [3] library. Table 6 summarizes the properties of each of the selected data sets, including the application domain, the number of total attributes $(|C|)$ and the number of total instances $(|U|)$. Each data set is divided into two categories: one for tree induction $(60 \%)$ and the other for calculating the average total cost for performance measurement by three tree induction algorithms, the CS-C4.5 algorithm, the CS-GainRatio algorithm, and the BDADT algorithm.

We run two groups of experiments and analyze their results in this section. The first group examines the effects of the BDA mechanism. In the second group, we compare our method with two previous approaches: the CS-C4.5 algorithm [11] and the CS-GainRatio algorithm [10].

These data sets have no test cost settings. The test costs often satisfy a normal distribution [28], which has been found to be applicable over most science and engineering measurements. Therefore, we use a normal distribution to generate random test costs in $[1,10]$. Meanwhile, the parameter $\theta$ ranges from -2 to -0.25 and the step size is 0.25 . In our experiments, all the selected data sets are randomly split into two subsets: a training data set (60\%) and a test data set $(40 \%)$. To facilitate processing and comparison, the values of conditional attributes are normalized to range from 0 to 1 in our experiments. We use $k=2$ in the misclassification cost matrix because the selected data sets all have two classes. The misclassification cost is defined as $m c=\left(\begin{array}{cc}0 & m c_{(0,1)} \\ m c_{(1,0)} & 0\end{array}\right)$, where $m c_{(1,0)}$ is the misclassification cost associated with a minority class object assigned to the majority class and $m c_{(0,1)}$ is the opposite misclassification scenario. For simplicity, we set $m c_{(0,1)}=\$ 50$ and $m c_{(1,0)}=10 m c_{(0,1)}=\$ 500$ in our experiments. 
In this section, we try to answer the following questions using two groups of experiments.

(1) Does the BDA mechanism assist in cost-sensitive decision tree construction?

(2) Is there an optimal setting of $\delta$ for any data set?

(3) Is the BDADT algorithm appropriate for minimal cost decision tree construction?

Table 6: Data sets used for the experiments.

\begin{tabular}{lllll}
\hline ID & Data Sets & Domain & $|C|$ & $|U|$ \\
\hline 1 & Biodeg & Chemical & 41 & 1055 \\
2 & Breast-w & Clinic & 9 & 699 \\
3 & Clean1 & Society & 166 & 476 \\
4 & ColonTumor & Clinic & 2000 & 62 \\
5 & Credit-a & Commerce & 15 & 690 \\
6 & Credit-g & Commerce & 20 & 1000 \\
7 & Diabetes & Clinic & 8 & 768 \\
8 & EEG-Eve State & Life & 14 & 1923 \\
9 & German & Society & 24 & 1000 \\
10 & Heart-statlog & Physics & 13 & 270 \\
11 & Ionosphere & Physics & 34 & 351 \\
12 & Magic04 & Physical & 10 & 19020 \\
13 & Promoters & Biology & 57 & 106 \\
14 & Prostate-GE & Clinic & 5966 & 102 \\
15 & SMK-CAN-187 & Society & 19993 & 187 \\
16 & Sonar & Physical & 60 & 208 \\
17 & Spam & Computer & 57 & 4601 \\
18 & Voting & Society & 16 & 435 \\
19 & Wdbc & Clinic & 30 & 569 \\
20 & Wpbc & Clinic & 33 & 198 \\
\hline
\end{tabular}

\subsection{Performance of the BDA mechanism}

To verify the performance of the BDA mechanism, we compare the results obtained by the BDADT algorithm for different values of $\delta$ on the 20 data sets. The algorithm generates a population of cost-sensitive decision trees for different $\delta$ values, which range from 0 to 1 with a step size of 0.25 . The value $\delta=0$ indicates the BDADT algorithm without the BDA mechanism. We generate 300 sets of test costs for each data set.

Figure 2 shows the average total costs obtained by the BDADT algorithm for different $\delta$ values on the 20 data sets under a normal distribution. From the results we observe the following.

(1) The BDA mechanism has good performance. As can be seen from Figure 2 , the average total costs obtained by the BDADT algorithm when $\delta=0$ are significantly larger than the average total costs obtained when $\delta$ takes other values on most of the data sets. 


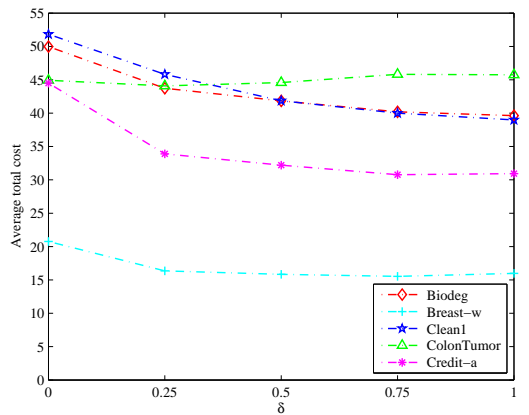

(a)

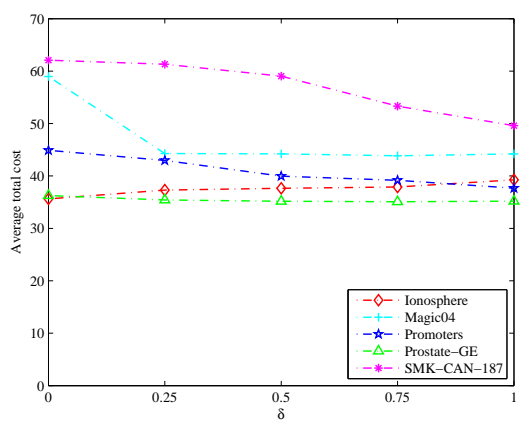

(c)

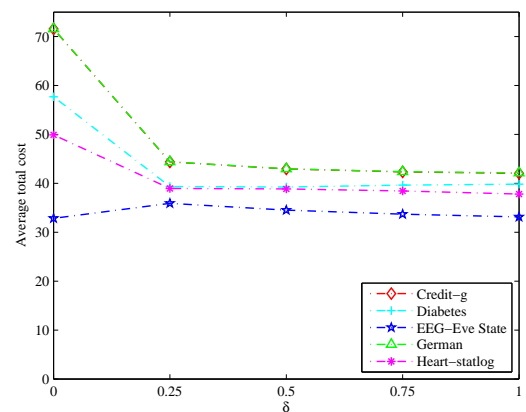

(b)

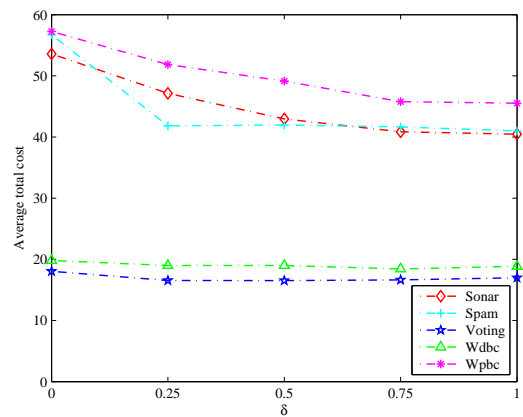

(d)

Figure 2: Comparison of average total costs obtained by BDADT algorithm with different $\delta$ settings on twenty data sets.

(2) As can be seen from Figures 2 a) and d), the average total costs decrease as $\delta$ increases for the Biodeg, Clean1, Sonar, and Wpbc data sets. That is, the BDA mechanism can reduce the interference of redundant attributes in decision tree construction.

(3) For the EEG-Eve State data set, the average total costs obtained are smallest when $\delta=0$. A possible reason for this is that the EEG-Eve State data set has a small number of attributes.

(4) There is no universally optimal value of $\delta$ for all of the data sets. From Figure 2, we see that the parameters in this algorithm are not sensitive for different data sets.

Figure 3 shows the running time of the BDADT algorithm for different $\delta$ values, where the unit for running time is millisecond. From the results for running time, we observe the following.

(1) The BDA mechanism is very efficient. It is obvious that the running times of the BDADT algorithm with $\delta=0$ are greater than those of the BDADT algorithm for other $\delta$ values for all 20 data sets that we selected in this experiment. 


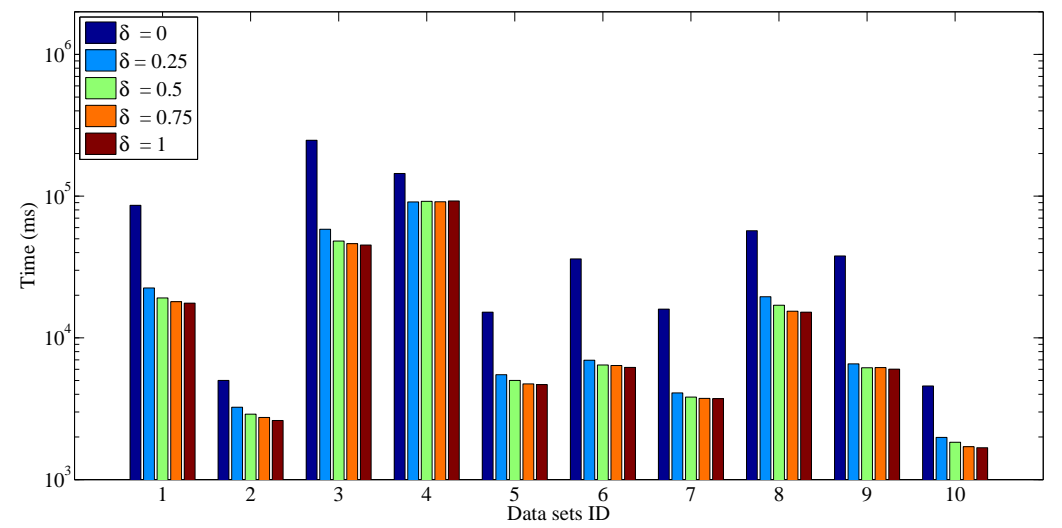

(a)

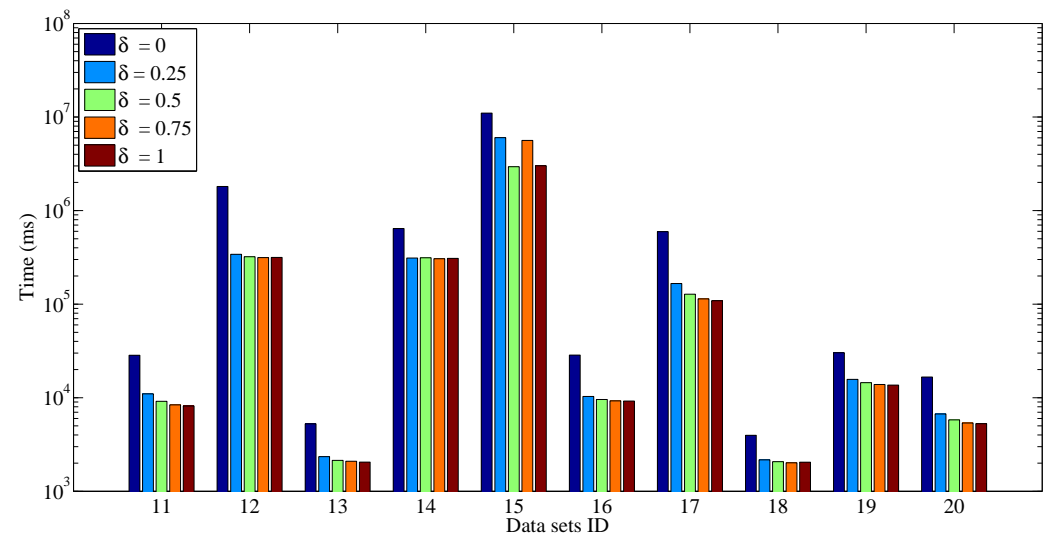

(b)

Figure 3: Comparison of the running time of BDADT algorithm by with different $\delta$ on twenty data sets.

(2) The running time of the BDADT algorithm decreases as the values of $\delta$ increase for most of the selected data sets. That is, as $\delta$ increases, more attributes are deleted in the process of node selection and the efficiency of the BDADT algorithm improves.

To summarize, the BDA mechanism is very effective. With the BDA mechanism, the total costs decrease for most of the data sets, as shown in Figure 2. The running time is also significantly reduced for all of the selected data sets, as shown in Figure 3. However, there is no universally optimal value of $\delta$. 


\subsection{Performance of the BDADT algorithm}

In this section, to demonstrate the effectiveness and efficiency of the BDADT algorithm, we conduct experiments on the selected data sets listed in Table 6. We experimentally compare the BDADT algorithm with the CS-C4.5 algorithm and the CS-GainRatio algorithm. For simplicity, we set $\delta=0.5$ in this experiment.

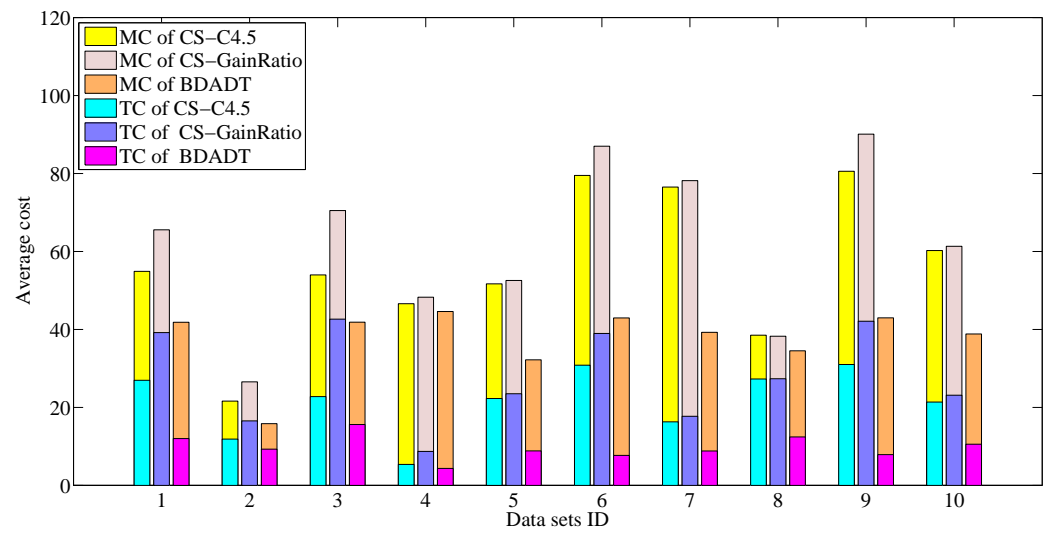

(a)

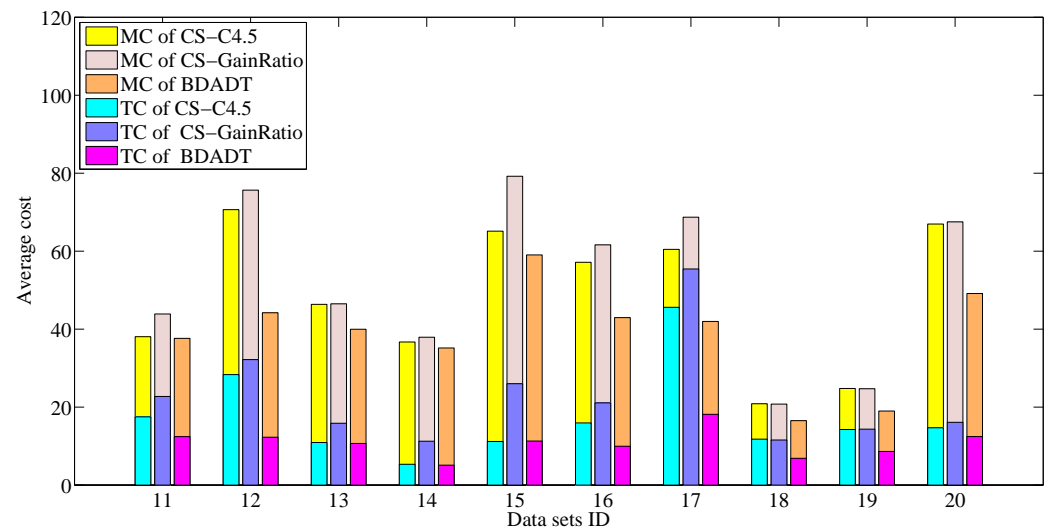

(b)

Figure 4: Comparison of three average costs between CS-C4.5, CS-GainRatio and BDADT algorithms on twenty data sets.

Figure 4 shows the average test cost (TC), the average misclassification cost (MC) and the average total cost (ATC) obtained by the three algorithms, which are CS-C4.5 algorithm, CS-GainRatio algorithm and BDADT algorithm. From Figure 4, we observe the following. 
(1) As can be seen from Figure 4, our proposed algorithm has the smallest average total cost for all 20 data sets. This means that the BDADT algorithm performs better than both the CS-C4.5 algorithm and the CS-GainRatio algorithm.

(2) The average test costs obtained by the BDADT algorithm are smaller than for other two algorithms in most cases. This means that many attributes are deleted in the process of node selection, and fewer tests are required.

(3) There is a trade-off between the test cost and the misclassification cost. For example, for the EEG-Eve State data set, the average misclassification cost obtained by the BDADT algorithm is larger than the average misclassification cost obtained by both the CS-C4.5 algorithm and the CS-GainRatio algorithm. However, the average total cost obtained by the BDADT algorithm is smaller than those obtained by the other two algorithms. As another example, for the Promoters data set, the average test cost obtained by the CS-C4.5 algorithm is smaller than that for the CS-GainRatio algorithm, but the average misclassification cost obtained by the CS-C4.5 algorithm is larger than that for the CS-GainRatio algorithm. Overall, these algorithms have nearly the same performance on this data set.

Figure 5 gives an intuitive understanding of the running time of the BDADT algorithm, the CS-C4.5 algorithm and the CS-GainRatio algorithm for the 20 data sets. From Figure 5, we have the following observations.

(1) The BDADT algorithm performs better than the CS-C4.5 algorithm and the CS-GainRatio algorithm. As can be seen from Figure 5, the running time of the BDADT algorithm is shorter than for the other two algorithms on all 20 data sets selected in this experiment.

(2) For the Biodeg, Clean1, Prostate-GE and SMK-CAN-187 data sets, the running time of the BDADT algorithm is significantly shorter than that of the CS-C4.5 algorithm and the CS-GainRatio algorithm.

(3) The proposed algorithm has greater efficiency for the data sets that have more condition attributes. For example, the running time of the proposed algorithm is $5094705 \mathrm{~ms}$ shorter than that of the CS-C4.5 algorithm on the SMK-CAN-187 data set, which has 19993 condition attributes. Meanwhile, the running time of BDADT is 6525 ms shorter than that of the CS-C4.5 algorithm on the Diabetes data set, which has eight condition attributes.

Table 7 lists the number of attributes deleted in the process of node selection for the 20 data sets. For simplicity, we list the first 10 nodes selected. The symbol "-" means that the decision tree construction has finished for this data set. From the information in Table 7, we observe the following. The number of deleted attributes decreases in the process of node selection in most cases. The most redundant attributes are deleted during root node selection, and the deleted ratios for the root node are listed in Table 8. The proposed algorithm largely reduces the calculations for the cost-sensitive tree construction. For instance, 13996 attributes are deleted when the root node is determined for the SMK-CAN-187 data set. In most cases, more than $50 \%$ of attributes are deleted in the process of root node selection. These attributes are not tested in the process of selecting later nodes. 


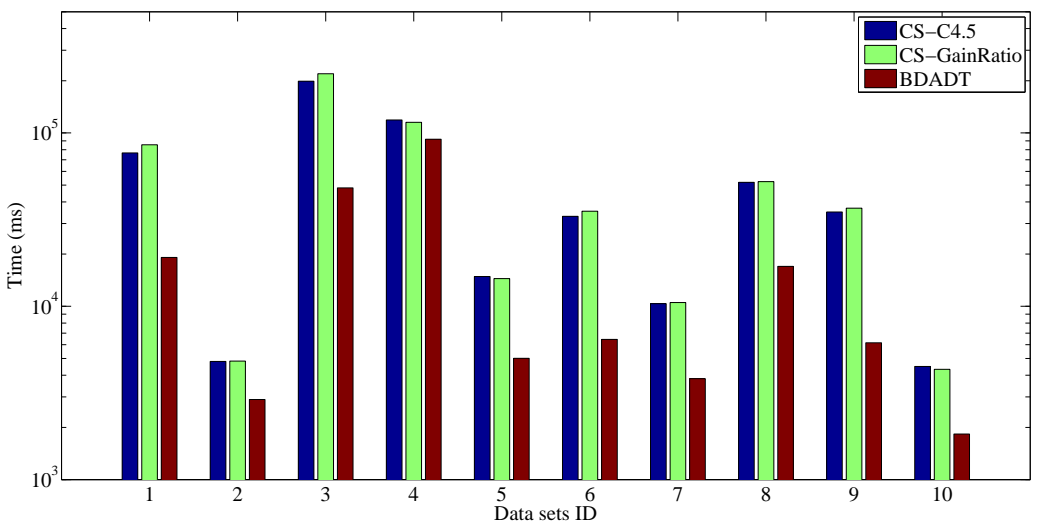

(a)

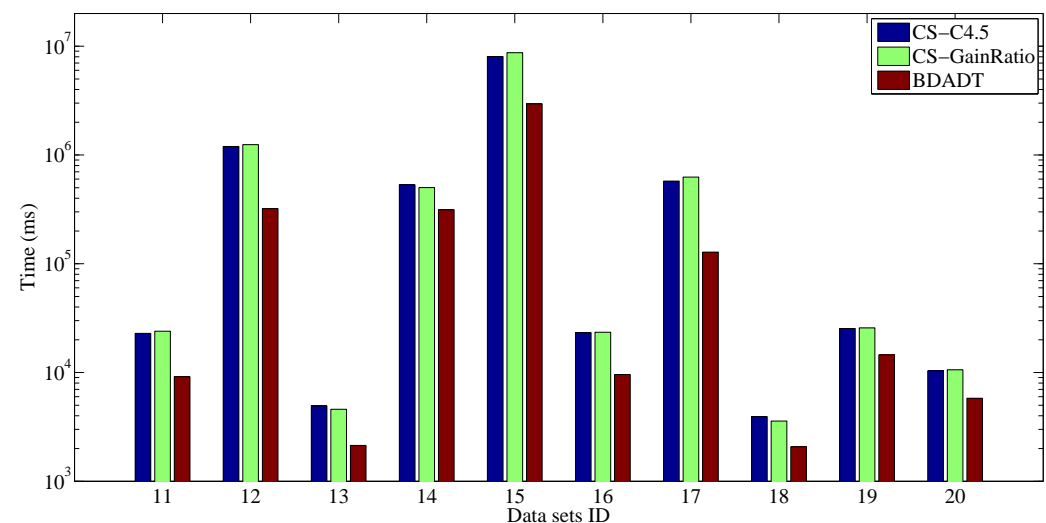

(b)

Figure 5: Comparison of the running time between CS-C4.5, CS-GainRatio and BDADT algorithms on twenty data sets.

From the above experiments, we observe the following. 1) The BDA mechanism is both effective and efficient. As can be seen from Figures 2 and 3, the average total costs obtained by the BDADT algorithm with the BDA mechanism are smaller than those without the BDA mechanism in most cases. Meanwhile, the BDADT algorithm with the BDA mechanism significantly reduces the running time for all data sets. Figure 3 shows this clearly. 2) The proposed algorithm performs well compared with the CS-C4.5 algorithm and the CS-GainRatio algorithm. Figures 4, 5 and Tables 7, 8 give us a detailed illustration. 
Table 7: Number of deleted attribute in first ten nodes selecting process.

\begin{tabular}{ccccccccccc}
\hline ID & \multicolumn{1}{c}{ Node } \\
\cline { 2 - 12 } & 1 & 2 & 3 & 4 & 5 & 6 & 7 & 8 & 9 & 10 \\
\hline 1 & 25 & 3 & 5 & 0 & 3 & 0 & 2 & 1 & 0 & 0 \\
2 & 4 & 1 & 1 & 0 & 0 & 0 & 0 & 1 & 1 & 0 \\
3 & 117 & 28 & 4 & 2 & 4 & 0 & 0 & 1 & 2 & 2 \\
4 & 1400 & 551 & 15 & 19 & 3 & 5 & - & - & - & - \\
5 & 5 & 1 & 2 & 0 & 0 & 2 & 1 & 0 & 0 & 2 \\
6 & 16 & 2 & 0 & 0 & 0 & 0 & 0 & - & - & - \\
7 & 4 & 0 & 1 & 1 & 1 & 0 & 0 & 0 & 0 & 0 \\
8 & 6 & 5 & 1 & 0 & 0 & 0 & 0 & 0 & 0 & 0 \\
9 & 17 & 3 & 0 & 2 & 0 & 1 & - & - & - & - \\
10 & 8 & 1 & 1 & 1 & 0 & 0 & 1 & 0 & 0 & 0 \\
11 & 24 & 1 & 2 & 1 & 1 & 0 & 0 & 0 & 0 & 1 \\
12 & 5 & 0 & 0 & 0 & 0 & 1 & 2 & 0 & 0 & 0 \\
13 & 40 & 9 & 6 & 0 & 1 & 0 & - & - & - & - \\
14 & 4773 & 1093 & 29 & 17 & 5 & 6 & 11 & 14 & - & - \\
15 & 13996 & 5898 & 4 & 4 & 45 & 24 & 2 & 3 & 1 & 4 \\
16 & 42 & 14 & 0 & 1 & 1 & 0 & 0 & 1 & 0 & 0 \\
17 & 40 & 2 & 1 & 1 & 2 & 0 & 2 & 0 & 4 & 0 \\
18 & 10 & 2 & 1 & 2 & 0 & 0 & 0 & 0 & - & - \\
19 & 21 & 3 & 1 & 1 & 1 & 0 & 0 & 0 & 0 & 0 \\
20 & 8 & 0 & 0 & 1 & 0 & 0 & 0 & 1 & 1 & 0 \\
\hline
\end{tabular}

Table 8: Ratio of deleted attribute in root node selecting process.

(a)

\begin{tabular}{|c|c|c|c|c|c|c|c|c|c|c|}
\hline \multirow{2}{*}{ Node } & \multicolumn{10}{|c|}{$I D$} \\
\hline & 1 & 2 & 3 & 4 & 5 & 6 & 7 & 8 & 9 & 10 \\
\hline root & $61 \%$ & $44 \%$ & $70 \%$ & $70 \%$ & $33 \%$ & $80 \%$ & $50 \%$ & $43 \%$ & $71 \%$ & $62 \%$ \\
\hline \multicolumn{11}{|c|}{ (b) } \\
\hline \multirow{2}{*}{ Node } & \multicolumn{10}{|c|}{$I D$} \\
\hline & 11 & 12 & 13 & 14 & 15 & 16 & 17 & 18 & 19 & 20 \\
\hline root & $71 \%$ & $50 \%$ & $70 \%$ & $80 \%$ & $70 \%$ & $70 \%$ & $70 \%$ & $63 \%$ & $70 \%$ & $24 \%$ \\
\hline
\end{tabular}

\section{Conclusion}

Minimal cost decision tree construction is an important task in machine learning. In this paper, we propose a cost-sensitive decision tree algorithm based on weighted class distribution with a BDA mechanism for this task. Our method uses a new heuristic function to select attributes that have low test cost and high information gain as nodes. Meanwhile, the BDA mechanism is used in the process of assigning nodes. Experiments on 20 data sets from the UCI Machine Learning Repository demonstrate that the effectiveness and feasibility of our approach. The experimental results show that the proposed algorithm is more effective and efficient than the existing CS-C4.5 and CSGainRatio algorithms. The minimal cost decision tree construction problem is important and challenging for medium or large data sets. Therefore, in the 
future, we aim to decrease the amount of calculation to speed up the algorithm.

\section{Acknowledgment}

This work is in part supported by the National Science Foundation of China under Grant Nos. 61379049 and 61379089.

\section{References}

[1] K. Alberti, P. Zimmet, Definition, diagnosis and classification of diabetes mellitus and its complications. part 1: diagnosis and classification of diabetes mellitus. provisional report of a who consultation, Diabetic Medicine 15 (7) (1998) 539-553.

[2] U. Alon, N. Barkai, D. A. Notterman, et al, Broad patterns of gene expression revealed by clustering analysis of tumor and normal colon tissues probed by oligonucleotide arrays, Proceedings of the National Academy of Sciences 96 (12) (1999) 6745-6750.

[3] A. Asuncion, D. Newman, Uci machine learning repository. university of california, school of information and computer science, irvine, ca (2007) (1994).

[4] J. Bobadilla, F. Ortega, A. Hernando, G. Glez-de Rivera, A similarity metric designed to speed up, using hardware, the recommender systems k-nearest neighbors algorithm, Knowledge-Based Systems 51 (2013) 27-34.

[5] H. Cekirge, I. Saatci, A new aneurysm occlusion classification after the impact of flow modification, American Journal of Neuroradiology 37 (1) (2016) 19-24.

[6] Y. Chen, C. Wu, K. Tang, Building a cost-constrained decision tree with multiple condition attributes, Information Sciences 179 (7) (2009) 967-979.

[7] Y. Chen, Q. Zhu, H. Xu, Finding rough set reducts with fish swarm algorithm, Knowledge-Based Systems 81 (2015) 22-29.

[8] W. Chine, A. Mellit, V. Lughi, A. Malek, G. Sulligoi, A. Pavan, A novel fault diagnosis technique for photovoltaic systems based on artificial neural networks, Renewable Energy 90 (2016) 501-512.

[9] G. Creamer, Y. Freund, Using boosting for financial analysis and performance prediction: application to s\&p 500 companies, latin american adrs and banks, Computational Economics 36 (2) (2010) 133-151.

[10] J. Davis, J. Ha, C. Rossbach, H. Ramadan, E. Witchel, Cost-sensitive decision tree learning for forensic classification, in: Machine Learning: European Conference on Machine Learning, Springer, 2006, pp. 622-629. 
[11] A. Freitas, A. Costapereira, P. Brazdil, Cost-sensitive decision trees applied to medical data, in: Data Warehousing and Knowledge Discovery, Springer, 2007, pp. 303-312.

[12] A. Gacek, W. Pedrycz, A granular description of ecg signals, IEEE Transactions on Biomedical Engineering 53 (10) (2006) 1972-1982.

[13] Q. He, Z. Xie, Q. Hu, C. Wu, Neighborhood based sample and feature selection for svm classification learning, Neurocomputing 74 (10) (2011) 1585-1594.

[14] Q. Hu, Y. Du, Z. Xie, Neighborhood classifiers, Expert Systems with Applications 32 (2) (2008) 866-876.

[15] Q. Hu, D. Yu, J. Liu, C. Wu, Neighborhood rough set based heterogeneous feature subset selection, Information Sciences 178 (18) (2008) 3577-3594.

[16] Q. Hu, L. Zhang, S. An, D. Zhang, D. Yu, On robust fuzzy rough set models, Fuzzy Systems, IEEE Transactions on 20 (4) (2012) 636-651.

[17] X. Jia, W. Liao, Z. Tang, L. Shang, Minimum cost attribute reduction in decision-theoretic rough set models, Information Sciences 219 (2013) 151167.

[18] S. Kanj, F. Abdallah, T. Denœux, K. Tout, Editing training data for multilabel classification with the k-nearest neighbor rule, Pattern Analysis and Applications 19 (1) (2016) 145-161.

[19] C. Kingsford, S. Salzberg, What are decision trees?, Nature Biotechnology 26 (9) (2008) 1011-1013.

[20] H. Leach, D. O'Connor, R. Simpson, H. Rifai, S. Mama, R. Lee, An exploratory decision tree analysis to predict cardiovascular disease risk in african american women, Health Psychology 35 (4) (2016) 397.

[21] J. Li, C. Mei, C. Kumar, X. Zhang, On rule acquisition in decision formal contexts, International journal of machine learning and cybernetics 4 (6) (2013) 721-731.

[22] J. Li, C. Mei, W. Xu, Y. Qian, Concept learning via granular computing: A cognitive viewpoint, Information Sciences 298 (2015) 447-467.

[23] X. Li, H. Zhao, W. Zhu, A cost sensitive decision tree algorithm with two adaptive mechanisms, Knowledge-Based Systems 88 (2015) 24-33.

[24] H. Liu, A. Gegov, M. Cocea, Rule based systems for big data: a machine learning approach, vol. 13, Springer, 2016.

[25] X. Ma, G. Wang, H. Yu, T. Li, Decision region distribution preservation reduction in decision-theoretic rough set model, Information Sciences 278 (2014) 614-640. 
[26] R. Mendez, L. Hake, T. Andresson, L. Littlepage, J. Ruderman, J. Richter, Phosphorylation of cpe binding factor by eg2 regulates translation of c-mos mrna, vol. 404, Nature Publishing Group, 2000.

[27] F. Min, Q. Liu, A hierarchical model for test-cost-sensitive decision systems, Information Sciences 179 (14) (2009) 2442-2452.

[28] F. Min, W. Zhu, A competition strategy to cost-sensitive decision trees, in: Rough Sets and Knowledge Technology, Springer, 2012, pp. 359-368.

[29] F. Min, W. Zhu, H. Zhao, Coser: cost-senstive rough sets (2016).

[30] S. Norton, Generating better decision trees, in: International Joint Conference on Artificial Intelligence, vol. 89, 1989.

[31] M. Ooi, H. Sok, Y. Kuang, S. Demidenko, C. Chan, Defect cluster recognition system for fabricated semiconductor wafers, Engineering Applications of Artificial Intelligence 26 (3) (2013) 1029-1043.

[32] Z. Pawlak, Rough sets and intelligent data analysis, Information Sciences 147 (1) (2002) 1-12.

[33] W. Pedrycz, Granular computing: Analysis and design of intelligent systems, CRC press, 2013.

[34] W. Pedrycz, R. Al-Hmouz, A. Saeed Balamash, A. Morfeq, Hierarchical granular clustering: An emergence of information granules of higher type and higher order, IEEE Transactions on Fuzzy Systems 23 (6) (2015) 22702283 .

[35] J. Quinlan, Simplifying decision trees, International Journal of Manmachine Studies 27 (3) (1987) 221-234.

[36] J. Quinlan, C4. 5: programs for machine learning, vol. 1, Morgan Kaufmann, 1993.

[37] A. Spira, J. E. Beane, V. Shah, et al, Airway epithelial gene expression in the diagnostic evaluation of smokers with suspect lung cancer, Nature Medicine 13 (3) (2007) 361-366.

[38] M. Tan, Cost-sensitive learning of classification knowledge and its applications in robotics, Machine Learning 13 (1) (1993) 7-33.

[39] P. Turney, Cost-sensitive classification: Empirical evaluation of a hybrid genetic decision tree induction algorithm, Journal of Artificial Intelligence Research (JAIR) 2 (1995) 369-409.

[40] P. Turney, Types of cost in inductive concept learning, Proceedings of the Cost-Sensitive Learning Workshop at the 17th ICML-2000 Conference (2000) 15-21. 
[41] T. Wang, Z. Qin, S. Zhang, C. Zhang, Cost-sensitive classification with inadequate labeled data, Information Systems 37 (5) (2012) 508-516.

[42] Y. Y. Yao, A partition model of granular computing, Lecture Notes in Computer Science (2004) 232-253.

[43] H. Yu, Z. Liu, G. Wang, An automatic method to determine the number of clusters using decision-theoretic rough set, International Journal of Approximate Reasoning 55 (1) (2014) 101-115.

[44] Z. Yu, Y. Liu, X. Yu, K. Pu, Scalable distributed processing of k nearest neighbor queries over moving objects, Knowledge and Data Engineering, IEEE Transactions on 27 (5) (2015) 1383-1396.

[45] N. Zavrtanik, J. Prosen, M. Tušar, G. Turk, The use of artificial neural networks for modeling air void content in aggregate mixture, Automation in Construction 63 (2016) 155-161.

[46] H. Zhao, F. Min, W. Zhu, Cost-sensitive feature selection of numeric data with measurement errors, Journal of Applied Mathematics 2013.

[47] H. Zhao, W. Zhu, Optimal cost-sensitive granularization based on rough sets for variable costs, Knowledge-Based Systems 65 (2014) 72-82.

[48] H. Zhao, Q. Hu, P. Wang, Cost-sensitive feature selection based on adaptive neighborhood granularity with multi-level confidence, Information Sciences 366 (2016) 134-149.

[49] W. Zhu, F. Wang, Reduction and axiomization of covering generalized rough sets, Information Sciences 152 (2003) 217-230. 